\title{
Perioperative Management of a Patient With Fontan Physiology for Posterior Spinal Fusion
}

\author{
Christian Walker ${ }^{\mathrm{a}}$, David Martin ${ }^{\mathrm{a}, \mathrm{b}}$, Jan Klamarc, Allan Beebe ${ }^{\mathrm{c}}$, Joseph D. Tobias ${ }^{\mathrm{a}, \mathrm{b}, \mathrm{d}, \mathrm{e}}$
}

\begin{abstract}
The Fontan operation remains a common palliative surgical approach for complex cardiac malformations characterized by single ventricle anatomy. Given improvements in surgical technique and perioperative care, these patients are now living into the second decade of life and beyond. As such, they may require additional surgical procedures to address other co-morbid conditions. We present a 12-year-old girl presenting for posterior spinal fusion for the treatment of scoliosis. She had been born with hypoplastic left heart syndrome and had subsequently undergone successful conversion to Fontan physiology. The authors describe the perioperative preparation and anesthetic management of the patient with Fontan anatomy.
\end{abstract}

Keywords: Fontan; Single ventricle; Scoliosis; Posterior spinal fusion; Congenital heart disease

\section{Introduction}

The Fontan operation was first performed in 1971 for the surgical repair of tricuspid atresia. Since then, the procedure

\footnotetext{
Manuscript accepted for publication May 15, 2014

${ }^{a}$ Department of Anesthesiology and Pain Medicine, The Ohio State University College of Medicine, Columbus, Ohio, USA

${ }^{\mathrm{b}}$ Department of Anesthesiology and Pain Medicine, Nationwide Children's Hospital, Columbus, Ohio, USA

'Department of Orthopedic Surgery, Nationwide Children's Hospital and The Ohio State University College of Medicine, Columbus, Ohio, USA

${ }^{\mathrm{d} D e p a r t m e n t}$ of Pediatrics, Nationwide Children's Hospital and The Ohio State University College of Medicine, Columbus, Ohio, USA

${ }^{\mathrm{e}}$ Corresponding Author: Joseph D. Tobias, Department of

Anesthesiology and Pain Medicine, Nationwide Children's Hospital,

700 Children's Drive, Columbus, Ohio 43205, USA.

Email: Joseph.Tobias@Nationwidechildrens.org

doi: http://dx.doi.org/10.14740/jmc1789w
}

has been used to correct various complex congenital cardiac malformations characterized by single ventricle physiology. The procedure connects vena caval blood directly to the pulmonary arteries (PAs) allowing the systemic venous blood to flow directly into the lungs. This allows the single ventricle to pump blood in series to both circulations, thereby alleviating chronic volume overload. Following the procedure, cardiac output (CO) is dependent not only on the function of the single ventricle, but also on a low pulmonary vascular resistance (PVR) to allow for passive pulmonary blood flow (PBF). We present a 12-year-old girl with Fontan anatomy who presented for posterior spinal fusion (PSF).

\section{Case Report}

IRB approval is not required for single-patient case reports at Nationwide Children's Hospital. The patient was a 12-yearold, $44.5 \mathrm{~kg}$ adolescent who was born with hypoplastic left heart syndrome (HLHS). She had undergone successful stage 1 and 2 palliation resulting in Fontan physiology. As the curve was progressing, the decision was made to proceed with surgical correction. During preoperative cardiac catheterization, three stents were placed (two in the left PA and one in the Glenn shunt) and the intra-atrial fenestration was closed. Echocardiogram 3 weeks prior to surgery showed normal RV function. Her medication regimen included enalapril $2.5 \mathrm{mg}$ twice a day, amitriptyline $2.5 \mathrm{mg}$ at bedtime and aspirin $81 \mathrm{mg}$ every day. Aspirin was discontinued 7 days prior to surgery. Laboratory evaluation 2 weeks prior to the procedure showed a hemoglobin of $14.8 \mathrm{~g} / \mathrm{dL}$, a hematocrit of $42.5 \%$ and a platelet count of $190,000 / \mathrm{mm}^{3}$. Coagulation studies were within normal limits. The patient arrived to the preoperative area from home on the morning of surgery with a room-air oxygen saturation of $97 \%$. She had been nil per $o s$ for $6 \mathrm{~h}$ except for her usual morning dose of enalapril. Following the administration of $50 \%$ nitrous oxide in oxygen for $3 \mathrm{~min}$, a 16-gauge intravenous cannula was placed. Anesthetic induction included etomidate $(0.2 \mathrm{mg} / \mathrm{kg})$, lidocaine $(1 \mathrm{mg} / \mathrm{kg})$ and fentanyl $(2 \mu \mathrm{g} / \mathrm{kg})$. Endotracheal intubation was facilitated by rocuronium $(0.5 \mathrm{mg} / \mathrm{kg})$. A second 16 intravenous cannula and a 20 -gauge radial arterial can- 
nula were placed. Using ultrasound guidance, a 4 French, 12-cm double-lumen catheter was placed in the right internal jugular vein. Additional monitoring included near infrared spectroscopy (NIRS) to monitor cerebral oxygenation and systemic oxygenation (deltoid muscle). Initial NIRS values varied from $65 \%$ to $80 \%$ in both sites. Anesthesia was maintained with desflurane to maintain the bispectral index at 50 - 60, a remifentanil infusion $(0.05-0.2 \mu \mathrm{g} / \mathrm{kg} / \mathrm{min})$ titrated to maintain hemodynamic stability, and a dexmedetomidine infusion $(0.2 \mu \mathrm{g} / \mathrm{kg} / \mathrm{h})$ without a bolus dose. Neurophysiological monitors were placed for intraoperative monitoring of motor and somatosensory evoked potentials. The patient was positioned prone and a forced air-warming blanket was placed. Prior to skin incision, cefazolin was administered for surgical prophylaxis. Tranexamic acid was administered (bolus dose of $50 \mathrm{mg} / \mathrm{kg}$ followed by an infusion of $5 \mathrm{mg} / \mathrm{kg} / \mathrm{h}$ ). Prior to the start of the surgical procedure, $500 \mathrm{~mL}$ of $5 \%$ albumin was administered to replace the fluid deficit and increased intravascular volume, and a milrinone infusion was started at $0.25 \mu \mathrm{g} / \mathrm{kg} / \mathrm{min}$. Initial arterial blood gas analysis was unremarkable with a venous saturation (blood obtained from the central venous catheter) of $70 \%$. Approximately 2 $\mathrm{h}$ into the case, there was moderate hypotension with a mean arterial pressure (MAP) of $50-52 \mathrm{~mm} \mathrm{Hg}$ instead of the goal MAP of $55-65 \mathrm{~mm} \mathrm{Hg}$. The hematocrit at that time was $28 \%$ and one unit of packed red blood cells was administered with the goal of maintaining the hematocrit $\geq 30 \%$. An additional $250 \mathrm{~mL}$ of $5 \%$ albumin was also administered. The duration of the surgical procedure was $140 \mathrm{~min}$. Prior to completion of the surgical procedure, acetaminophen (15 $\mathrm{mg} / \mathrm{kg})$ and hydromorphone $(0.01 \mathrm{mg} / \mathrm{kg})$ were administered for postoperative analgesia. Ondansetron $(0.15 \mathrm{mg} / \mathrm{kg})$ was administered to prevent postoperative nausea and vomiting. The remifentanil infusion and desflurane were discontinued and the patient was turned to the supine position. The dexmedetomidine and milrinone infusions were continued. The patient's trachea was extubated in the operating room and she was transported to the cardiac intensive care unit. Total blood loss was estimated at $800 \mathrm{~mL}$. Total fluids included 1,200 $\mathrm{mL}$ of isotonic crystalloid, $750 \mathrm{~mL}$ of $5 \%$ albumin, $193 \mathrm{~mL}$ of donor PRBCs and $250 \mathrm{~mL}$ of intraoperative cell salvage. Postoperative analgesia was provided by patient-controlled analgesia with hydromorphone. Intravenous acetaminophen was administered every $6 \mathrm{~h}$ for the initial 48 postoperative hours. The milrinone and dexmedetomidine infusions were continued until the next morning when her home medication regimen was resumed. Hemoglobin and hematocrit reached a postoperative low of $10.9 \mathrm{~g} / \mathrm{dL}$ and $31.5 \%$ respectively on postoperative day 1 . The patient was transferred to the inpatient floor on postoperative day 1 and discharged to home on postoperative day 5. On follow-up with the orthopedic service at 3 months, the patient was noted to have intact neurologic and vascular function. Radiographic imaging revealed correction of the scoliosis to $24^{\circ}$ with intact instrumentation.

\section{Discussion}

The Fontan procedure has undergone several modifications and has been applied to a variety of complex cardiac malformations characterized by a single functional ventricle including HLHS. By connecting the venous return from the superior and inferior vena cava directly to the pulmonary arteries, the Fontan circulation allows the single ventricle to pump blood in series to the systemic and pulmonary circulations thereby alleviating chronic volume overload. The Fontan operation is currently divided into two separate surgical stages. The first stage, known as a bidirectional Glenn shunt or the hemi-Fontan, connects the SVC to the PA. During the second stage with the connection of the IVC to the PA, the Fontan circulation is completed [1].

Scoliosis has been reported to have an increased incidence following either sternotomy or thoracotomy [2]. When patients with Fontan anatomy require surgical intervention, the perioperative care is impacted by the primary type of $\mathrm{CHD}$ as well as the anesthetic implications of the surgical procedure. Given the complex nature of our patient's CHD, close collaboration with our pediatric cardiology colleagues was required. This included not only echocardiography, but also cardiac catheterization with electrophysiology evaluation. During these investigations, interventions (stent placement and closure of the intra-atrial fenestration) were determined. The latter intervention was chosen to prevent a paradoxical embolism during a surgical procedure in which venous air embolism may occur. The preoperative evaluation of our patient revealed normal RV function and normal PVR, two of the components necessary for successful function of the Fontan circulation [3].

PSF may result in significant intraoperative blood loss, at times in excess of an entire blood volume. This may be exacerbated by the presence of abnormalities of coagulation function in patients with cyanotic CHD [4]. Preoperative coagulation function should be evaluated and treatment with vitamin $\mathrm{K}$ or blood products instituted as needed. Multiple techniques to minimize intraoperative blood loss and allogeneic transfusions can be employed during major orthopedic procedures including autologous blood donation, acute normovolemic hemodilution, intraoperative blood salvage, controlled hypotension, and manipulation of the coagulation cascade with DDAVP, anti-fibrinolytic agents (epsilon-aminocaproic acid, tranexamic acid and aprotinin) or recombinant factor VIIa [5]. In our clinical practice, the commonlyused techniques include controlled hypotension, tranexamic acid as an anti-fibrinolytic agent and intraoperative cell salvage. Given the concerns regarding cardiac function during periods of controlled hypotension, tissue oxygenation with near infrared spectroscopy was employed to ensure adequate cardiac output intraoperatively [6]. The placement of a CVP allowed for monitoring of lactate and venous oxygen saturation intraoperatively [7]. 
Table 1. Perioperative Care of the Patient With Fontan Anatomy During Posterior Spinal Fusion

\section{Reference number Outcome}

[15]

\begin{abstract}
14-year-old boy with Fontan physiology undergoing PSF. Preoperative testing included a trial of anesthetized positive pressure ventilation and position changes without surgical intervention. TIVA to facilitate neurophysiological monitoring. Intraoperative monitoring included arterial cannula, CVP and TEE. Significant hypotension associated with decreased ventricular filling on TEE with no change in CVP. EBL was 1,200 mL. Allogeneic blood transfusion was minimized by using autologous blood donation and intraoperative blood salvage.
\end{abstract}

15-year-old boy with Fontan physiology. EBL in excess of 3.5 L, requiring significant intraoperative resuscitation with blood and blood products. This report highlights the potential for significant blood loss in these patients and discusses options to limit this complication.

Retrospective cohort of seven patients with Fontan physiology for PSF. Average blood loss was 2,942 $\mathrm{mL}$ (range: 1,100 - 7,500). Swan-Ganz catheters were used for monitoring. Complication rate of $85.7 \%$ including superior mesenteric artery syndrome, acute renal tubular necrosis, pleural effusion requiring chest tube placement, urinary tract infection, Horner syndrome, and a late case of paralysis at $48 \mathrm{~h}$.

Two adolescents with Fontan physiology. One patient had a pacemaker prohibiting using of motor evoked potentials. The patient experienced ventricular fibrillation requiring cardiopulmonary resuscitation during a wake-up test. The authors theorized that the wake-up test may constitute an increase in cardiac work load that may prove intolerable to patients with palliated congenital heart disease.

Reported the use of TEE to titrate intraoperative dopamine and epinephrine infusions. Suggested the use of TEE in addition to standard monitors.

Retrospective analysis of eight patients with Fontan physiology for PSF. Average EBL was $1,520 \mathrm{~mL}$. Although all of the patients required intraoperative blood transfusion, $62 \%$ avoided allogeneic products through autologous donation. Postoperative complications occurred in three patients including one death related to hypovolemic shock refractory to treatment and two patients with pleural effusions requiring chest tube placement. The authors stressed the need for a multi-disciplinary team, a thorough evaluation of the patient's cardiac status and a low threshold for TEE.

PSF: posterior spinal fusion; TIVA: total intravenous anesthesia; CVP: central venous pressure; TEE: trans-esophageal echocardiography; EBL: estimated blood loss.

Patients remain at risk for thromboembolic events after the Fontan procedure with thromboembolic events being the second leading cause of late death [8]. As such the majority of patients receive some time of chronic prophylaxis with either coumadin or aspirin. Given the limited evidence-based medicine regarding the risk-benefit ratio of stopping aspirin therapy for more than 7 days, our pediatric cardiologists continue to recommend its administration up to that time [9].

To reduce afterload and minimize RV strain our patient was chronically receiving enalapril. Severe hypotension refractory to adrenergic agonists under general anesthesia can be associated with inhibition of the angiotensin converting enzyme (ACE) pathway. This effect can be magnified by on- going intraoperative blood loss. Hypotension refractory to adrenergic agents may be responsive to vasopressin therapy [10]. Patients that continue their ACE inhibitors may have a lower incidence of hypertension in the postoperative period which would help limit strain on the RV in the Fontan physiology and improve cardiac output. These risks must be assessed by the individual provider and careful assessment of the patient's cardiac status should be taken into account when deciding whether to continue ACE inhibitors in the perioperative period.

The general goals of intraoperative care include blunting of the surgical stress response and obtaining an acceptable plane of anesthesia while allowing for early tracheal extuba- 
tion with minimal effects on PVR. Key to the intraoperative care is avoidance of agents with significant negative inotropic effects. Given these concerns, we chose an intravenous induction using a combination of etomidate and fentanyl. Etomidate has been shown in multiple studies to have stable hemodynamic properties in both adult and pediatric patients [11]. The adrenal suppressive effects of etomidate due to its inhibition of 11- $\beta$ hydroxylase are well known although the perioperative implications of this effect have not been clearly demonstrated $[12,13]$. Regardless of the technique being used, skilled airway management during anesthetic induction remains more important than the technique itself [14]. Following anesthetic induction and endotracheal intubation, the anesthetic is tailored to blunt the sympathetic stress response, control PVR and provide amnesia and analgesia while allowing for early tracheal intubation. We chose a technique that incorporated the synthetic opioid, remifentanil, desflurane and dexmedetomidine. This would not only provide adequate intraoperative anesthesia, but also blunt the sympathetic stress response and allow for early tracheal extubation. Furthermore, the use of this regimen provides for rapid awakening such changes be noted on neurophysiological monitoring and a wake-up test be requested.

During the postoperative period, the management focuses on maintaining adequate myocardial function of the single ventricle and preventing increases in PVR [14]. General management to maintain to avoid changes in PVR includes maintaining normothermia, normocarbia, normoxia and a normal $\mathrm{pH}$. Additionally, adequate control of pain while limiting the deleterious effects of opioids on ventilator function is required. Phosphodiesterase inhibitors such as milrinone, which was used in our patient, may not only improve myocardial performance, but also serve to dilate the pulmonary vascular bed. If these measures fail to adequately control PVR, the addition of inhaled nitric oxide (NO) may be considered.

Pain-free, spontaneous ventilation generates a negative interpleural pressure thereby enhancing hemodynamic performance with Fontan physiology. Inappropriate or early tracheal extubation may lead to hypoventilation and associated hypercarbia, hypoxemia and atelectasis, all of which can lead to an increase in PVR. Opioids and benzodiazepines may cause respiratory depression making early tracheal extubation difficult in post-Fontan patients. Dexmedetomidine, a highly selective $\alpha_{2}$-adrenergic receptor agonist, has been shown to offer advantages over commonly-used regimens including opioids and benzodiazepines. Furthermore, adjunctive agents including acetaminophen or non-steroidal anti-inflammatory agents should be incorporated into the postoperative analgesic regimen, given their opioid sparing effects.

Previous authors have reported anecdotal experiences with the perioperative care of this population during PSF (Table 1) [15-20]. The Fontan operation, while provid- ing a corrective procedure for complex cardiac malformations characterized by single ventricle anatomy, leaves the patient's CO dependent on passive PBF. Therefore, careful perioperative management centered on maintaining a passive PBF by keeping the PVR low and preload high is critical to maintaining adequate cardiac output in these patients. The effective care of all these patients necessitates the involvement of a multi-disciplinary team.

\section{References}

1. Feinstein JA, Benson DW, Dubin AM, Cohen MS, Maxey DM, Mahle WT, Pahl E, et al. Hypoplastic left heart syndrome: current considerations and expectations. J Am Coll Cardiol. 2012;59(1 Suppl):S1-42.

2. Herrera-Soto JA, Vander Have KL, Barry-Lane P, Myers JL. Retrospective study on the development of spinal deformities following sternotomy for congenital heart disease. Spine (Phila Pa 1976). 2007;32(18):1998-2004.

3. Gewillig M. The Fontan circulation. Heart. 2005;91(6):839-846.

4. van Nieuwenhuizen RC, Peters M, Lubbers LJ, Trip MD, Tijssen JG, Mulder BJ. Abnormalities in liver function and coagulation profile following the Fontan procedure. Heart. 1999;82(1):40-46.

5. Tobias JD. Strategies for minimizing blood loss in orthopedic surgery. Semin Hematol. 2004;41(1 Suppl 1):145156.

6. Tobias JD. Cerebral oxygenation monitoring: nearinfrared spectroscopy. Expert Rev Med Devices. 2006;3(2):235-243.

7. Walley KR. Use of central venous oxygen saturation to guide therapy. Am J Respir Crit Care Med. 2011;184(5):514-520.

8. Khairy P, Fernandes SM, Mayer JE, Jr., Triedman JK, Walsh EP, Lock JE, Landzberg MJ. Long-term survival, modes of death, and predictors of mortality in patients with Fontan surgery. Circulation. 2008;117(1):85-92.

9. Smart S, Aragola S, Hutton P. Antiplatelet agents and anaesthesia. Contin Educ Anaesth Crit Care Pain. 2007; 7:157-161.

10. Wheeler AD, Turchiano J, Tobias JD. A case of refractory intraoperative hypotension treated with vasopressin infusion. J Clin Anesth. 2008;20(2):139-142.

11. Tobias JD. Etomidate: applications in pediatric critical care and pediatric anesthesiology. Pediatr Crit Care Med. 2000;1(2):100-106.

12. Komatsu R, You J, Mascha EJ, Sessler DI, Kasuya Y, Turan A. Anesthetic induction with etomidate, rather than propofol, is associated with increased 30-day mortality and cardiovascular morbidity after noncardiac surgery. Anesth Analg. 2013;117(6):1329-1337.

13. Wagner CE, Bick JS, Johnson D, Ahmad R, Han X, 
Ehrenfeld JM, Schildcrout JS, et al. Etomidate use and postoperative outcomes among cardiac surgery patients. Anesthesiology. 2014;120(3):579-589.

14. Gaca JA, Douglas WI, Barnes SD. Anesthetic implications of the Fontan procedure for single ventricle physiology. Semin CardiothoracVasc Anesth. 2001;5:31-39.

15. Vischoff D, Fortier LP, Villeneuve E, Boutin C, Labelle $H$. Anaesthetic management of an adolescent for scoliosis surgery with a Fontan circulation. Paediatr Anaesth. 2001;11(5):607-610.

16. Rafique MB, Stuth EA, Tassone JC. Increased blood loss during posterior spinal fusion for idiopathic scoliosis in an adolescent with Fontan physiology. Paediatr Anaesth. 2006;16(2):206-212.

17. Hedequist DJ, Emans JB, Hall JE. Operative treatment of scoliosis in patients with a Fontan circulation. Spine (Phila Pa 1976). 2006;31(2):202-205.

18. Leichtle CI, Kumpf M, Gass M, Schmidt E, Niemeyer T. Surgical correction of scoliosis in children with congenital heart failure (Fontan circulation): case report and literature review. Eur Spine J. 2008;17(Suppl 2):S312317.

19. Farid IS, Reddy A, Lewis J, Kendrick EJ. Continuous intraoperative TEE monitoring for a child with Fontan pathway undergoing post spinal fusion. Middle East J Anesthesiol. 2013;22(1):109-112.

20. Perez-Caballero Macarron C, Sobrino Ruiz E, Burgos Flores J, Vazquez Martinez J, Coca Perez A, Alvarez Rojas E, Sanchez Ruas J. Spinal surgery in the univentricular heart--is it viable? Cardiol Young. 2014;24(1):73-78. 